beet tissue had no effect, however. The active substance has not yet been separated from the waterextract.

The presence of an inhibiting substance in beetroot during storage accounts for the delay in the commencement of both cation and anion absorption from solutions for about fifty hours after cutting the intact roots. The delay can be shortened by washing the cut tissue and so removing the inhibitor. It must be assumed that the substance will also disappear from tissue by a natural process, probably of a respiratory nature, since when disks are kept in a limited volume of solution they eventually begin to absorb salts. The inhibiting substance is thought to be concerned with the natural dormancy of beetroot during the winter, because extracts of young freshly pulled roots and old stored roots in the early summer do not inhibit salt absorption.

The results will be reported fully elsewhere.

W. J. REES

A. D. SkELDing

Botany Department,

University, Birmingham 15. Sept. 14.

\section{Incidence of Salmonellæ in Fæces of Dogs suffering from Distemper}

Cruickshank and Williams Smith $^{1}$ found five of five hundred healthy dogs (1 per cent) in London to be excreting salmonellæ in their fæces. The technical methods used in this investigation consisted, briefly, in plating the samples directly on to Leifson's agar, and also after preliminary enrichment in selenite $F$ medium and in tetrathionate broth. Wolff, Henderson and $\mathrm{McCallum}^{2}$ examined frcal specimens from a hundred city dogs in Michigan by preliminary incubation in tetrathionate broth followed by plating on S.S. agar. They found 18 (18 per cent) to be positive for salmonellæ. Most of the positive samples were obtained from dogs suffering from distemper or from dogs maintained in a kennel where outbreaks of distemper and enteritis had occurred in the six months previous to sampling. Kintner ${ }^{3}$, simply by direct plating on S.S. agar, found 13 of 71 dogs (18 per cent) brought to the Ohio State Veterinary Clinic for treatment to be excreting salmonellæ in their frees. Of the dogs in this investigation, 50 per cent of those suffering from distemper were positive for salmonellæ, as were 10 per cent of the non-distemper cases.

In view of the marked difference between the American and British figures, it was decided to in vestigate the problem further. With the help of a number of veterinary surgeons practising in the London area, rectal swabs were examined from a hundred dogs suffering from distemper or paradistemper (hard pad disease). The methods employed were those used by Cruickshank and Williams Smith1. Only one of the samples (1 per cent) was found to contain salmonellæ. This organism, Salm. typhimurium, was isolated after preliminary enrichment in selenite $F$ medium.

These results suggest that there is little or no difference between the incidence of salmonellæ in the fæces of normal and distemper-infected dogs in London, and that distemper alone does not account for the incidence of salmonellæ in the fæces of dogs in American cities being so much higher than those in London. Kintner ${ }^{3}$ considers that the high incidence in American dogs may be related to the diet, and quotes references to show the very high percentage of $\mathrm{dog}$ foods which may contain salmonellæ. Wolff et al. ${ }^{2}$ also noted that some of the dogs examined by them were fed on reject eggs, and that the salmonellæ isolated from many of those dogs were of the 'dried-egg' variety. It is possible, therefore, that the difference between the American and British figures may be accounted for by a difference in diet.

H. Williams Smith

A. Buxton

Animal Health Trust,

Houghton Grange,

Houghton, Huntingdon. July 7.

${ }^{1}$ Cruickshank, J. C., and Williams Smith, H., Brit. Med. J., ii, 1254 (1949).

2 Wolff, A. H., Henderson, N. D., and McCallum, G. L., Amer. J. Pub. Hith., 38, 403 (1948).

3 Kintner, I., Vet. Med., 44, 396 (1949).

\section{Planck's Constant and the Fine-Structure Constant}

Advances in fundamental theory have often been guided by the principle that reliance is to be placed on the most directly observed quantities, that is, those involving least theoretical assumptions. This letter indicates the interpretations of Planck's constant $(h)$ and of the fine-structure constant $(\alpha)$ which follow from this principle ${ }^{1}$.

Of the measurements on which quantum theory is based, the most direct are lengths (wave-lengths, path curvatures). As is well known, the three classical theories of gravitation, electron dynamics, and the electromagnetic field are each scale-free, there being no constant of the dimensions of a length in their laws, taken separately. But the quantum theory of discrete eigenstates is scale-fixed, $h$ providing in combination with other constants (for example, in the combinations $h / m c, h^{2} / 4 \pi^{2} m e^{2}$, and $h^{3} c / 2 \tau m e^{4}$, where $m$ equals electron mass) the basic standards of linear scale required to represent these states. Apart from heat effects, which involve less direct measurements and more assumptions, $h$ is always calculated from characteristic lengths or from ratios of lengths. Thus the above principle suggests that from the point of view of a future theory the characteristic feature of the phenomena involving $h$ is their definiteness of scale, rather than the 'quantization' of action or energy. (Lengths of the order $e^{2} / m c^{2}$ are less reliable than the above lengths involving $h$, for they are less directly measured and rest on a high extrapolation of the inverse square law from larger-scale observations.)

The most direct measurements yielding $\alpha$ involve ratios of the above three lengths (ratios of characteristic wave-lengths, and of these to atomic radii) or of wave-lengths to path curvatures (for example, in the photo-electric effect). Our principle therefore requires $\alpha$ to be interpreted as the ratio of fundamental lengths, as has already been proposed ${ }^{2}$ on less general grounds. It follows that a derivation of $\alpha$ must show how assumptions containing explicitly or implicitly one fundamental length, and no arbitrary pure numbers determinable only by experiment, can account for the presence in current theory of several fundamental lengths; compare Heisenberg's idea ${ }^{3}$ that "the introduction of a fundamental length may be bound up with a new fundamental modification of the formalism". 